\title{
Rule Britannia
}

Eye (2005) 19, 727-728. doi:10.1038/sj.eye.6701645

The study by Johnston and colleagues, entitled Pilot National Electronic Cataract Surgery Survey, published in this month's edition is as unassuming in its title as it is revolutionary in its implications. To the best of my knowledge, this work represents the first demonstration of potential for pooling the surgical results from an entire region or country in an on-line database in order to learn how best to care for patients.

Over the past several decades, ophthalmologic researchers (as have clinical researchers in general) have shifted from anecdotal case reports and series to various methodologies for gathering data in a manner that represents the population of people about whom inferences will be drawn. Those methodologies include randomized prospective studies, broad outcomes analyses, crosssectional studies, and claims data analysis. I have been an investigator in all of those methodologies and have grappled with the advantages and considerable disadvantages of each. The technology described by Johnston and colleagues, if implemented broadly and efficiently has the potential to both combine and eclipse all of these technologies.

Clearly, randomized prospective trials are viewed as having the best potential to answer specific clinical questions, generally with regard to treatment superiority. However, they suffer from the highest expense associated with performance. Moreover, it is nearly impossible to conduct a clinical trial in which subjects and their level of illness are representative of those in the underlying population. Similarly, for surgical clinical trials, the surgeons who participate are frequently a highly selected crowd.

Some useful clinical insights can be obtained by 'outcome studies,' a term that has been increasingly abused in the decade since colleagues and I published the first studies in the ophthalmologic literature to use that appellation. As originally intended, the notion was to sample the clinical results obtained in a sufficiently broad sample of the population as to
JC Javitt

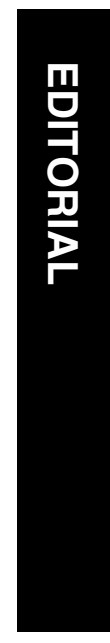

be representative of the results typically obtained in community practice. In the context of our work, we randomly selected surgeons from national lists, stratified by their surgical volume and sampled sequential patients within their practices for study. In recent years, the term 'outcomes study' has been erroneously applied to case series from one or a few institutions that are attempting to make their work appear as something other than the analysis of a convenience sample of patients. Even under the best of circumstances, however, data collected using the manual techniques we employed in 1990 are inherently less reliable than those collected through a real-time system such as Johnston's where the data collected for patient care and for clinical research are one and the same.

In an attempt to gain the broadest possible national sample in order to learn the 'truth' about rates of complications of surgery, such as endophthalmitis, retinal detachment, and similar rare events, I have in the past been associated with analyses of administrative data sets in the United States, Denmark, and elsewhere. These studies provided interesting findings and benchmark data. However, nobody assumes that the administrative data are coded with perfect accuracy. One only hopes that errors in coding are equivalent in cases and controls for a sample of several million people. Such was the case, when we were challenged to restudy a claims-data-based analysis that showed a correlation between Nd:YAG capsulotomy and subsequent retinal detachment. The original finding was considered so inflammatory that a repeat study was commissioned, this time with funds to obtain primary information from both the cataract surgeon and the retinal surgeon. As we expected, the coding was imperfect. Some patients coded as having had cataract surgery had not, some patients coded as having had retinal detachments did not have them, and some patients coded as having had an uncomplicated postoperative course had, indeed suffered complications. Nevertheless, as we expected, the coding errors were equally
Washington, DC, USA

Correspondence: JC Javitt Washington, DC, USA

E-mail: jjavitt@ healthdirections.net

Dr Javitt is Executive Vice President of First Consulting Group, Inc., and Chief Scientific Advisor to Active Health Management, Inc. In 2004, he was appointed by President Bush to the President's Information Technology Advisory Committee, where he chairs the Health Subcommittee. He continues to serve as an Adjunct Senior Fellow of the Potomac Institute for Policy Studies (Arlington, VA) and an Adjunct Professor of Ophthalmology at the Johns Hopkins School of Medicine (Baltimore, MD). He is the author of the first book on Computers in Medicine and has combined a career in academic ophthalmology with an ongoing involvement with the health information technology industry. He can be contacted at jjavitt@ healthdirections.net. 
distributed among the Nd:YAG patients and those who did not undergo capsulotomy. Thus, the finding of a fourfold increased risk of cataract surgery was shown in the second study, exactly as it was in the first. I remember the week it was published being asked by a

distinguished professor of ophthalmology whether I felt embarrassed about the number of coding errors uncovered in my earlier work. From my perspective, I was surprised neither that the coding was imperfect, nor that the result was proven all over again despite the imperfect coding.

The methodology described by Johnston and coworkers has the potential to turn all of that upside down. The UK has embarked on the most ambitious national health computerization project in the world. In fact, I have been involved in exchanges between its leaders and our own. By taking proactive steps to include collection of ophthalmologic data at the outset, ophthalmology distinguishes itself as a proactive specialty committed to clinical excellence. Moreover, ophthalmology gains the upper hand in defining how data about the eye and its disorders will be recorded and maintained, rather than waiting for that to be defined by others much less familiar with eye disease.
A national framework such as that described by Johnston has the potential to make clinical trials recruitment and data capture a far less onerous and expensive task. Moreover, this framework enables the prospect of clinical trials in which not only treatment allocation, but recruitment itself is conducted randomly. Today, we are faced with questions that defy our ability to fund and organize clinical trials. A perfect example is whether clear-cornea, sutureless cataract wounds are more commonly associated with endophthalmitis than traditional wounds. A clinical trial to answer that question will require more than 50000 patients in each group. Even if recruitment and data collection costs could be brought down to $\$ 100$ per person (typical costs are $\$ 1000$ to $\$ 5000$ per person) the cost of the trial is extremely high. In a system such as that described by Johnston, such a question could be answered on an outcomes basis essentially for free and on a random treatment allocation basis at costs far lower than are conceivable in any other environment.

If the prototype system that Johnston describes is implemented successfully on a national basis, the UK is likely to be the gold standard for population-wide understanding of eye disease and the outcomes of treating it for quite some time. 